\title{
COMMUNICATION
}

\section{Imputabilité médicamenteuse des réactions lichénoïdes selon l'OMS.}

\author{
Masson Regnault E1,2, Catros S, Brunet S, Marteau JM, Fricain JC \\ 1. Hôpital Pellegrin - CHU Bordeaux \\ 2. Profession libérale
}

\section{Introduction}

Les réactions lichénoïdes iatrogènes, ou médicamenteuses sont caractérisées par des lésions similaires au lichen plan. L'imputabilité médicamenteuse est conditionnée par plusieurs critères : délai d'apparition des lésions, disparition des lésions après retrait du traitement, ré-introduction positive, description dans la littérature de cas similaires.

Le but de notre étude était de recenser les réactions lichénoïdes d'origine médicamenteuse en confrontant la littérature à la méthode d'imputabilité de l'OMS.

\section{Matériels et méthodes}

Une recherche bibliographique sur le moteur de recherche Pubmed en utilisant les mots clefs : lichenoid reaction AND/OR lichenoid drug AND/OR lichenoid drug eruption AND/OR lichenoid de 1944 à 2016. Le recensement des cas publiés a été complété par une analyse de la bibliographie des articles retenus. Tous les articles recensant et décrivant des cas de $\mathrm{RL}$ d'origine médicamenteuse ont été retenus. Les cas publiés rapportant une réaction lichénoïde d'origine médicamenteuse muqueuse et/ou cutanée ont été classé dans un tableau selon différents critères d'imputabilité : Réaction cutanée et/ou muqueuse ; histologie ; description sémiologique ; disparition des lésions à l'arrêt du traitement ; réintroduction positive ; test allergique ; facteur favorisant ; autres explications. Tous les rapports de cas ou les articles de revue pour lesquels les critères d'imputabilité nécessaires n'étaient pas rapportés ont été exclus du tableau car ils ne permettaient pas d'appliquer une méthode d'imputabilité.

\section{Résultat}

115 références bibliographiques sont retenus sur 288. 149 patients sont rapportés sur les articles sélectionnés. Un examen histologique a été réalisé chez $82 \%$ des patients. Notre revue de la littérature nous a rapporté 88 molécules dont l'imputabilité est fortement suspectée sans quelle puisse être affirmé. 17 molécules sur ces 88 sont reconnues par le Vidal comme molécules pouvant engendrer l'apparition de réaction lichénoïde. $90 \%$ des réactions lichénoïdes sont observées dans la première année du traitement.

\section{Conclusion}

La prévalence des réactions lichénoïdes d'origine médicamenteuse est probablement sous-estimée et la découverte de lésion de lichen plan chez un patient doit amener le clinicien à mener une enquête médicamenteuse - date d'apparition des lésions, date d'introduction d'un nouveau traitement, analyse de la littérature.

emmanuel.masson.regnault@gmail.com

(C) The authors, published by EDP Sciences. This is an Open Access article distributed under the terms of the Creative Commons Attribution License 4.0 (http://creativecommons.org/licenses/by/4.0/). 